\title{
A Case of Old Age-Onset Generalized Pustular Psoriasis with a Deficiency of IL-36RN (DITRA) Treated by Granulocyte and Monocyte Apheresis
}

\author{
Chiharu Tominaga Masaaki Yamamoto Yasutomo Imai \\ Kiyofumi Yamanishi \\ Department of Dermatology, Hyogo College of Medicine, Nishinomiya, Japan
}

\section{Key Words}

Pustular psoriasis · Psoriasis vulgaris · IL-36RN · Mutation · Granulocyte and monocyte apheresis · Cytokines

\begin{abstract}
A 78-year-old woman who had been suffering from psoriasis vulgaris for 31 years was admitted to hospital because of her erythroderma. A toxic eruption was suspected and she was treated with prednisolone $30 \mathrm{mg}$ daily. However, it was ineffective and, suspecting psoriatic erythroderma, cyclosporine $150 \mathrm{mg}$ daily was administered with tapering of the prednisolone. Two weeks after a dose reduction of cyclosporine to $100 \mathrm{mg} /$ day, erythroderma with widespread generalized pustules and fever developed. Histology of a biopsy revealed inflammatory infiltrates in the skin with a spongiform pustule of Kogoj, which was consistent with generalized pustular psoriasis (GPP). Her pustules improved with additional etretinate $20 \mathrm{mg} /$ day, but the erythroderma persisted and she consulted us. Three sessions of granulocyte and monocyte apheresis once weekly were effective for her condition and decreased her serum levels of IL-6 and IL-8. She had homozygous mutations of c.[28C $>$ T] in IL36RN which cause p.[Arg10Ter]. She is the oldest reported case of GPP with a deficiency of interleukin-36 receptor antagonist (DITRA), although GPP in DITRA has been suggested to usually occur in younger cases with no pre-existing psoriasis vulgaris.

(C) 2015 S. Karger AG, Basel
\end{abstract}

KARGER 125/s $\begin{aligned} & \text { Kiyofumi Yamanishi, MD, PhD } \\ & \text { Department of Dermatology, Hyogo College of Medicine } \\ & \text { 1-1, Mukogawa-cho, Nishinomiya, Hyogo, 663-8501 (Japan) } \\ & \text { E-Mail kyamanis@hyo-med.ac.jp }\end{aligned}$


Case Reports in
Dermatology

\begin{tabular}{l|l}
\hline \multicolumn{2}{l}{ Case Rep Dermatol 2015;7:29-35 } \\
\hline DOI: 10.1159/000380876 & $\begin{array}{l}\text { ○ 2015 S. Karger AG, Basel } \\
\text { www.karger.com/cde }\end{array}$ \\
\hline
\end{tabular}

Tominaga et al.: A Case of Old Age-Onset Generalized Pustular Psoriasis with a Deficiency of IL-36RN (DITRA) Treated by Granulocyte and Monocyte Apheresis

\section{Introduction}

Generalized pustular psoriasis (GPP) is an inflammatory skin disorder which occurs in patients with or without psoriasis vulgaris and is characterized by sudden fevers and extensive erythemas with pustules and edema, sometimes with life-threatening circulatory and/ or respiratory disturbances. Those symptoms develop recurrently and therefore lifelong treatment is often required to manage disease activity. Mutations in $I L 36 R N$ have been identified in familial GPP and in sporadic cases of GPP $[1,2]$. Cases with IL-36RN mutations have been named as deficiency of IL-36RN (DITRA) [1]. Recently, two other genes, CARD14 and $A P 1 S 3$, have also been found to be responsible for GPP $[3,4]$.

The efficacy of granulocyte and monocyte apheresis (GMA) on GPP has been investigated [5] and GMA was indicated for GPP in Japan in October 2012. For treatment of GPP, vitamin A analogs, cyclosporine, methotrexate and TNF- $\alpha$ inhibitors are available, but in some cases with GPP those drugs are not necessarily effective or applicable because they are pediatric cases or involve patients that are elderly, pregnant or have hepatitis B virus infections. GMA may be used for such cases as an alternative or an adjuvant therapy to those medications. Here, we report a 78-year-old patient, the oldest-onset case of GPP ever reported with DITRA, who was successfully treated by GMA.

\section{Case Presentation}

A 78-year-old woman complained of erythroderma and was hospitalized. She had been suffering from psoriasis vulgaris since 47 years of age and used over-the-counter drugs for treatment. She had hypertension and chronic heart failure which were managed with acetazolamide, candesartan cilexetil and amlodipine besylate. Her brother had psoriasis vulgaris and her niece had psoriatic arthritis. Her daughter had arthritis with a high anti-nuclear antibody (ANA) titer $(\times 1,240)$, but her diagnosis was not established (fig. 1a). A toxic eruption was suspected for the patient and $30 \mathrm{mg}$ daily prednisolone was administered. However, it was ineffective, and the dose of prednisolone was decreased and instead cyclosporine $150 \mathrm{mg}$ daily was given for 12 days. Cyclosporine was effective and the dose was decreased to $100 \mathrm{mg}$ daily, but 2 weeks later, a sudden fever, generalized edema, erythroderma with multiple pustules and lakes of pus occurred (fig. 2a). A skin biopsy showed infiltrates of neutrophils into the upper spinous and subcorneal layers of the epidermis which formed a Kogoj spongiform pustule (fig. 2b). The diagnosis of GPP was made and etretinate $20 \mathrm{mg}$ daily was administered. The pustules subsided but the fever and erythroderma with edema continued, so she was referred to us.

On examination, edematous generalized erythroderma with exudations and scales was seen, but pustules were absent (fig. 1b, left). A blood test showed a white blood cell count of $7,380 / \mu \mathrm{l}$ (segment $78.5 \%$, lymphocytes $15.3 \%$, monocytes $6.0 \%$, eosinophils $0.1 \%$, basophils $0.1 \%$ ), and a high C-reactive protein level of $13.5 \mathrm{mg} / \mathrm{dl}$. The severity score for GPP [6] was 12 (skin score 6 plus score for general symptoms and blood tests 6). She was treated with bed rest and a combination of cyclosporine and etretinate for 1 week, but the GPP score remained at 10. For supportive therapy, GMA was introduced once weekly. After the initial session of GMA, the white blood cell and C-reactive protein levels began to decrease and edema, skin redness and scales became less severe. Before GMA, the neutrophil cell count was 4,500 cells/ $\mu \mathrm{l}$, but after three sessions of GMA, neutrophils had decreased to 1,608 cells/ $\mu$ l. At 6 days after the last GMA, the severity score for GPP had improved to 5 (fig. $1 \mathrm{~b}$, 
Tominaga et al.: A Case of Old Age-Onset Generalized Pustular Psoriasis with a Deficiency of IL-36RN (DITRA) Treated by Granulocyte and Monocyte Apheresis

center). Further sessions of GMA were held, and neutrophils recovered to normal levels by 19 days, at which time the GPP severity score was 4 (fig. 1b, right).

During hospitalization, the patient complained of a dry mouth feeling and had a positive ANA titer $(\times 320)$ and a high titer of anti-SSA and anti-SSB auto-antibodies. Sjögren syndrome was diagnosed and the symptoms were controlled by cevimeline hydrochloride. After treatment with GMA, the dose of cyclosporine was decreased and the drug was eventually discontinued, with no exacerbation of GPP. She was followed up with etretinate $10 \mathrm{mg}$ daily and topical vitamin D3 and steroids for the remaining lesions of psoriasis vulgaris. Relapse of GPP has been absent for 2 years to date.

The IL36RN gene was examined under approval of the Institutional Review Boards and informed consent from the patient and her daughter. PCR products from their genomic DNAs were subjected to Sanger sequencing. Genetic analysis revealed homozygous mutations c. [28C >T];[28C >T] which led to p.[Arg10Ter];[Arg10Ter] in the patient (fig. 3a). Her daughter had a heterozygous mutation c.[28C >T]; [=] in IL36RN (fig. 3b). The HLA types of the patient were HLA-A*0201, HLA-B*0201, HLA-DRB1*0803 and HLA-DRB1*1405.

The serum cytokine levels of IL-1 $\beta$, IL-6, IL- 8 and TNF- $\alpha$ were examined at the patient's first visit, 1 day before the first session of GMA, 2 days after the second session of GMA and 19 days after the last session of GMA (fig. 4). Those cytokine levels had already decreased before the first session of GMA. The serum levels of IL- 6 and IL-8 further decreased along with the improvement of the GPP score by GMA, but the levels of IL- $1 \beta$ and TNF- $\alpha$ did not.

\section{Discussion}

Since the discovery of $I L 36 R N$ as a gene responsible for GPP, at least part of the disease is recognized as an inheritable inflammatory skin disorder. Mutations of $I L 36 R N$ have now been reported in more than 70 cases of GPP worldwide. The mutations of c. [28C $>\mathrm{T}]$ and c. [115+6T $>C$ C] are predominant in Japanese cases with DITRA [7] and possibly those are founder mutations. The present case also had homozygous mutations c.[28C $>\mathrm{T}] ;[28 \mathrm{C}>\mathrm{T}]$ which lead to premature termination of p.[Arg10Ter];[Arg10Ter], and thereby IL-36RN is inactivated. The age of onset of GPP ranged from 2 to 68 years in previously reported cases with DITRA, and $65 \%$ of those were $<30$ years old. Pre-existing psoriasis vulgaris was documented in $65 \%$ of those cases with DITRA. In some of those cases with psoriasis vulgaris, an association with HLA-A*0206 has been suggested [7], but that did not apply to the present case. GPP in DITRA occurs at early ages [8] and in cases without plaque-type psoriasis [7]. The present patient is not comparable to those reports because of the old-age onset and the precedent psoriasis vulgaris. The patient's daughter also had a heterozygous c.[28C $>\mathrm{T}]$ in IL36RN. Heterozygous mutations are found in cases with GPP [7], and therefore she might be at risk of developing GPP.

The present case developed psoriasis vulgaris at the age of 47 , and it became psoriatic erythroderma at 78 years of age along with an exacerbation of heart failure, which caused an $18 \mathrm{~kg}$ body weight gain with generalized edema. She had dental caries, and the onset of GPP was 1 week after a tooth had been extracted. Drugs, dental and upper respiratory infections, pregnancy and solar irradiation may be precipitating factors for GPP [9]. In the present case of DITRA, physical stress, such as the invasive treatment of a dental extraction and/or the aggravation of heart failure, might have been triggering factors for GPP.

The present case was treated by GMA along with cyclosporine and etretinate, because those drugs were not so effective for erythroderma and generalized edema of GPP, and TNF- $\alpha$ inhibitors were avoided due to her old age and heart failure. GMA is a therapeutic 
Tominaga et al.: A Case of Old Age-Onset Generalized Pustular Psoriasis with a Deficiency of IL-36RN (DITRA) Treated by Granulocyte and Monocyte Apheresis

apheresis that selectively removes activated leukocytes by an extracorporeal system using a column of cellulose acetate beads (Adacolumn ${ }^{\circledR}$; Japan Immunoresearch Laboratories Co., Ltd., Takasaki, Japan). Plasma immunoglobulins and complement iC3b bind to cellulose acetate beads and then granulocytes and monocytes are adsorbed to the beads via the $\mathrm{Fc} \gamma \mathrm{R}$ and/or iC3b receptor 3 (CR3) (MAC-1) expressed on the cell surface [10]. GMA is used for treatment of inflammatory bowel disease (IBD) and its efficacy for treating GPP has been recently reported [5]. However, the precise therapeutic mechanism for GPP has not been fully elucidated. Andoh et al. [11] have shown that blood levels of IL-1 $\beta$, TNF- $\alpha$, IL- 6 and IL-8 were decreased by five sessions of GMA in 4 cases with IBD, and they suggested that the suppression of those inflammatory cytokines may be involved in the therapeutic efficacy of GMA. In the present case with GPP, those cytokine levels began to decrease with the GPP score before GMA, possibly because of the bed rest and treatment with cyclosporine and etretinate. The levels of IL- 8 and IL- 6 were responsive to GMA in our case, with a decrease in the GPP score from 10 to 5 by the three sessions of GMA. However, in contrast with those IBD cases, TNF- $\alpha$ and IL- $1 \beta$ levels did not reflect the efficacy of GMA and the severity of GPP in our patient.

The efficacy of GMA in DITRA with mutation of c.[28C $>\mathrm{T}]$; [28C $>\mathrm{T}]$ has been recently reported in a GPP case without psoriasis vulgaris, in whom five sessions of GMA improved pustules and edema [12]. The present case shows that GMA is also effective as a supportive therapy for GPP and for an old age-onset case in DITRA with psoriasis vulgaris.

\section{Acknowledgements}

The authors thank members of the Joint-Use Research Facilities of the Hyogo College of Medicine for their helpful technical assistance. This work was supported in part by JSPS KAKENHI grants number 25860972 and 26461668.

\section{Disclosure Statement}

The authors declare no conflicts of interest.

\section{References}

1 Marrakchi S, Guigue P, Renshaw BR, Puel A, Pei XY, Fraitag S, et al: Interleukin-36-receptor antagonist deficiency and generalized pustular psoriasis. N Engl J Med 2011;365:620-628.

-2 Onoufriadis A, Simpson MA, Pink AE, Di Meglio P, Smith CH, Pullabhatla V, et al: Mutations in IL36RN/IL1F5 are associated with the severe episodic inflammatory skin disease known as generalized pustular psoriasis. Am J Hum Genet 2011;89:432-437.

-3 Jordan CT, Cao L, Roberson ED, Pierson KC, Yang CF, Joyce CE, et al: PSORS2 is due to mutations in CARD14. Am J Hum Genet 2012;90:784-795.

4 Setta-Kaffetzi N, Simpson MA, Navarini AA, Patel VM, Lu HC, Allen MH, et al: AP1S3 mutations are associated with pustular psoriasis and impaired Toll-like receptor 3 trafficking. Am J Hum Genet 2014;94:790-797.

-5 Ikeda S, Takahashi H, Suga Y, Eto H, Etoh T, Okuma K, et al: Therapeutic depletion of myeloid lineage leukocytes in patients with generalized pustular psoriasis indicates a major role for neutrophils in the immunopathogenesis of psoriasis. J Am Acad Dermatol 2013;68:609-617.

-6 Umezawa Y, Ozawa A, Kawasima T, Shimizu H, Terui T, Tagami H, et al: Therapeutic guidelines for the treatment of generalized pustular psoriasis (GPP) based on a proposed classification of disease severity. Arch Dermatol Res 2003;295(suppl 1):S43-S54. 
Tominaga et al:: A Case of Old Age-Onset Generalized Pustular Psoriasis with a Deficiency of IL-36RN (DITRA) Treated by Granulocyte and Monocyte Apheresis

7 Sugiura K, Takemoto A, Yamaguchi M, Takahashi H, Shoda Y, Mitsuma T, et al: The majority of generalized pustular psoriasis without psoriasis vulgaris is caused by deficiency of interleukin-36 receptor antagonist. J Invest Dermatol 2013;133:2514-2521.

-8 Sugiura K, Takeichi T, Kono M, Ogawa Y, Shimoyama Y, Muro Y, et al: A novel IL36RN/IL1F5 homozygous nonsense mutation, p.Arg10X, in a Japanese patient with adult-onset generalized pustular psoriasis. Br J Dermatol 2012;167:699-701.

$>9$ Farber EM, Nall L: Pustular psoriasis. Cutis 1993;51:29-32.

10 Hiraishi K, Takeda Y, Shiobara N, Shibusawa H, Jimma F, Kashiwagi N, et al: Studies on the mechanisms of leukocyte adhesion to cellulose acetate beads: an in vitro model to assess the efficacy of cellulose acetate carrier-based granulocyte and monocyte adsorptive apheresis. Ther Apher Dial 2003;7:334-340.

11 Andoh A, Tsujikawa T, Inatomi O, Deguchi Y, Hata K, Kitoh K, et al: Suppression of inflammatory cytokine secretion by granulocyte/monocyte adsorptive apheresis in active ulcerative colitis. Ther Apher Dial 2005;9:123-127.

12 Sugiura K, Haruna K, Suga Y, Akiyama M: Generalized pustular psoriasis caused by deficiency of interleukin-36 receptor antagonist successfully treated with granulocyte and monocyte adsorption apheresis. J Eur Acad Dermatol Venereol 2014;28:1835-1836.

a

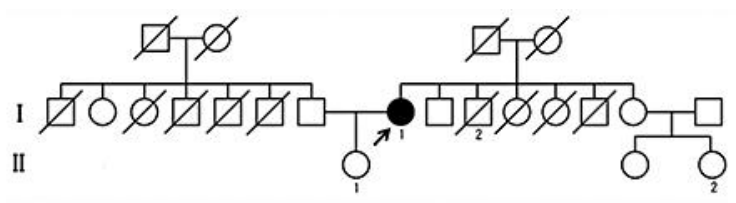

$\mathbf{b}$
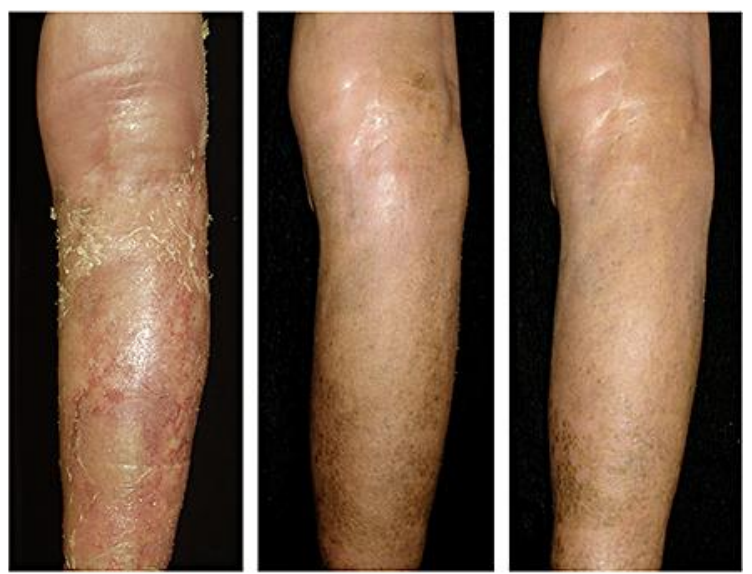

Before GMA

6 days

19 days

After GMA

Fig. 1. a Pedigree of the case. The present case (I-1) had had psoriasis vulgaris for 31 years and developed GPP at 78 years of age. Her brother (I-2) had psoriasis vulgaris and her daughter (II-1) had arthritis with a high titer of ANA. Her niece (II-2) had psoriatic arthritis. b Clinical course of the lesion on her right lower leg before and after GMA. Left: The patient showed diffuse edema and severe erythema and desquamations at her first visit, 1 week before treatment with GMA. The severity score of GPP was 12 . Center: Edema, erythemas and scales were markedly improved at 6 days after three sessions of GMA. The severity score was 5. Right: The skin conditions were well controlled at 19 days after the last GMA session. The severity score was 4 . 


\section{Case Reports in Dermatology}

\begin{tabular}{l|l}
\hline DOI: $10.1159 / 000380876$ & $\begin{array}{l}\text { C 2015 S. Karger AG, Basel } \\
\text { www.karger.com/cde }\end{array}$ \\
\hline
\end{tabular}

Tominaga et al.: A Case of Old Age-Onset Generalized Pustular Psoriasis with a Deficiency of IL-36RN (DITRA) Treated by Granulocyte and Monocyte Apheresis
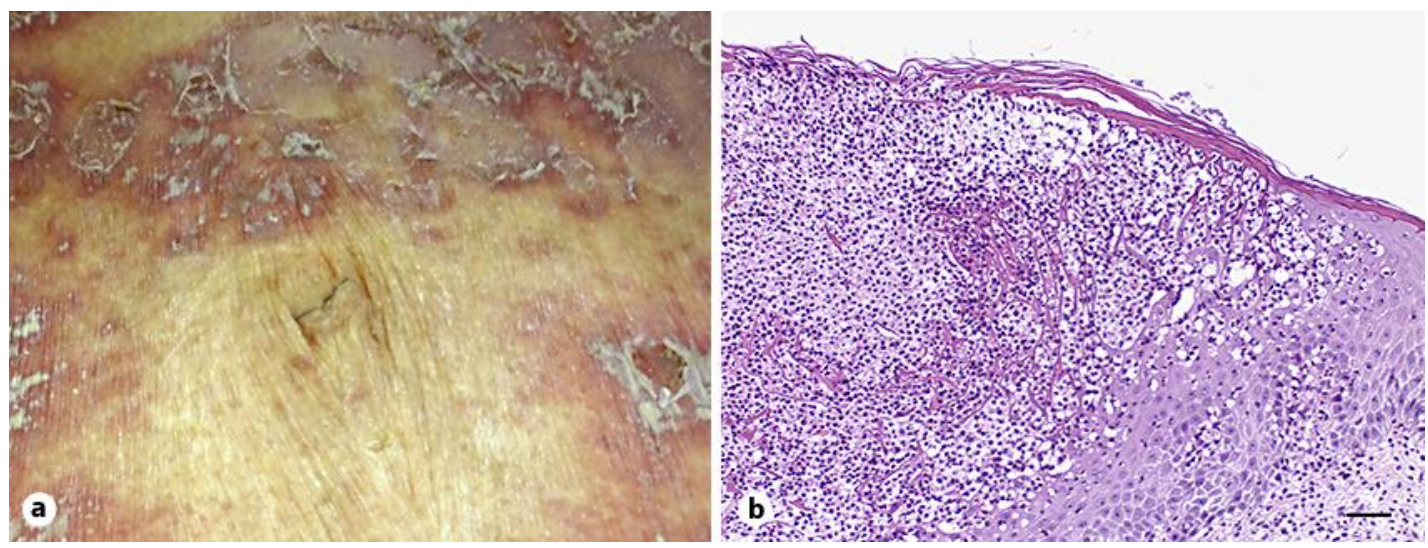

Fig. 2. a Erythemas with multiple pustules and pus lakes at the onset of GPP. b Histology of a lesion. Infiltrates of neutrophils into the epidermis and a spongiform pustule of Kogoj are evident. Scale bar $=50 \mu \mathrm{m}$.

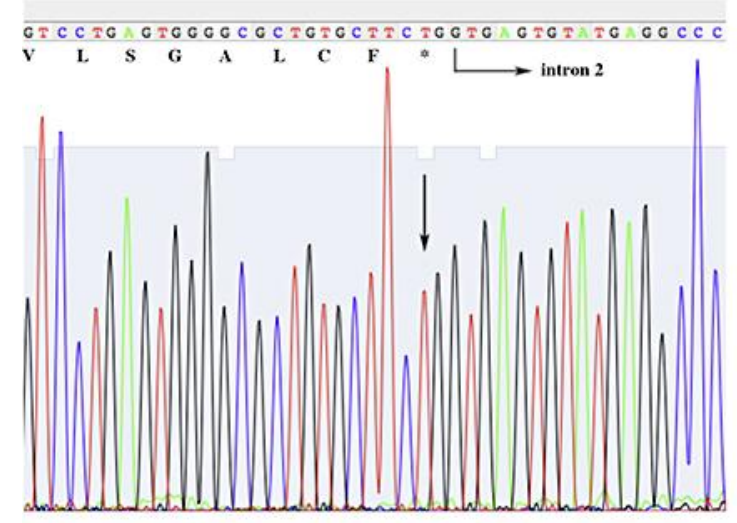

a

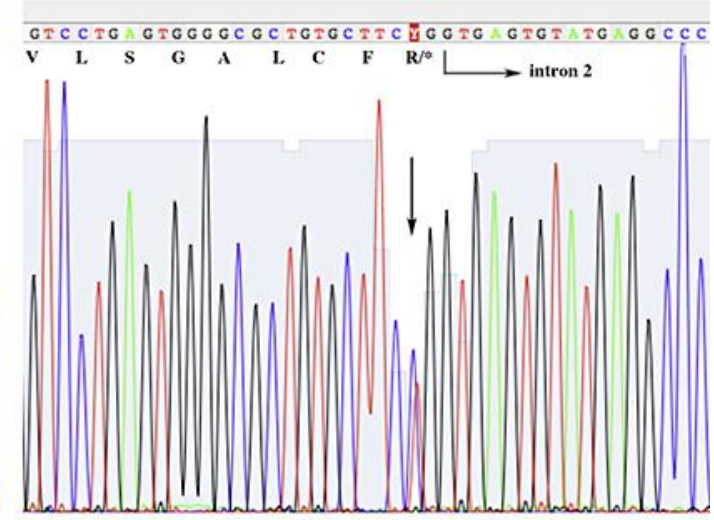

b

Fig. 3. IL-36RN mutations of the present case (a) and her daughter (b). Arrows indicate each mutation of c. [28C >T];[28C >T] (a) and c. [28C >T];[=] (b), respectively, in IL36RN. 
Tominaga et al:: A Case of Old Age-Onset Generalized Pustular Psoriasis with a Deficiency of IL-36RN (DITRA) Treated by Granulocyte and Monocyte Apheresis

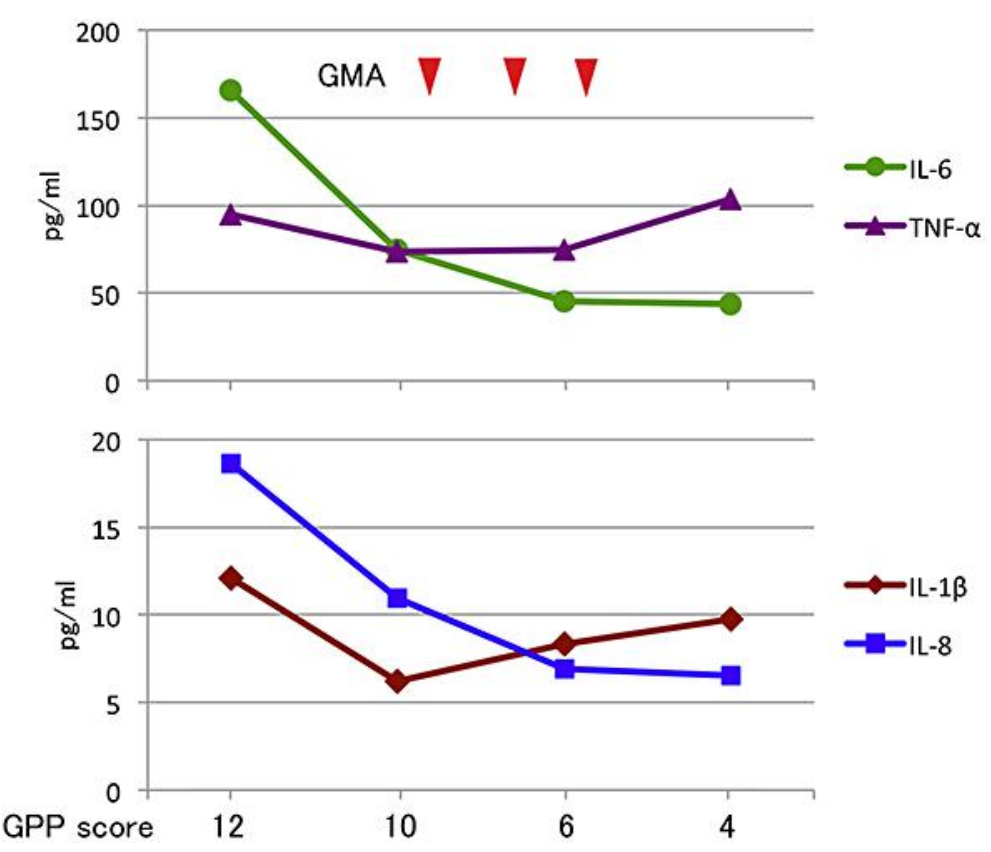

Fig. 4. Serum cytokine levels in the course of the case. Blood was sampled 1 week and 1 day before the first session of GMA, 2 days after the second session of GMA and 19 days after the last session of GMA. Concentrations of serum cytokines, IL-6, TNF- $\alpha$, IL-1 $\beta$ and IL-8 were measured using Bio-Plex Pro human cytokine multiplex assay kits (Bio-Rad, Hercules, Calif., USA) and a Bio-Plex 200 System with highthroughput fluidics (Bio-Rad). The severity score of GPP for each day is indicated. The serum levels of IL-1 and IL-6 decreased with the improvement of GPP. 